Original Paper http://ajol.info/index.php/ijbcs http://indexmedicus.afro.who.int

\title{
Part contributive de la densité démographique au reverdissement de certaines zones fortement anthropisées du Sahel : cas des Communes d'Aguié et d'Ibohamane au Niger
}

\author{
Salifou SAIDOU ${ }^{1 *}$ et Jean-Marie Karimou AMBOUTA ${ }^{2}$ \\ ${ }^{I}$ Ecole Doctorale de Sciences de la Vie et de la Terre, Faculté d'Agronomie, Université Abdou Moumouni de \\ Niamey, BP : 10960 Niamey, Niger. \\ ${ }^{2}$ Département Sciences des Sols, Faculté d'Agronomie, Université Abdou Moumouni de Niamey. BP : 10960 \\ Niamey, Niger. \\ *Auteur correspondant ; E-mail : saidousalifou29@yahoo.fr, Tel (+227) 96962406
}

\section{RESUME}

Cette étude porte sur l'analyse de la dimension humaine dans l'explication du reverdissement observé dans le paysage de certaines zones fortement anthropisées du Sahel. A cet effet, la commune d'Aguié et celle d'Ibohamane ont été choisies en prenant en compte leur forte densité démographique. La méthodologie utilisée repose sur l'application d'une analyse corrélative couplée à des tests statistiques sur les valeurs de pluies annuelles, les densités démographiques et les valeurs de l'indice normalisé de végétation sur la série chronologique 1980-2018. Les résultats obtenus mettent en évidence deux séquences pluviométriques sèche et humide statistiquement significatives. Pour autant, la période humide récente bien installée à partir de la décennie 90 n'explique pas en totalité l'expression du reverdissement de ces zones. Des taux de $60 \%$ et $45 \%$ de ce changement sont associés à la densité démographique respectivement au niveau de la commune d'Aguié et celle d'Ibohamane. Ces résultats permettent de replacer le rôle central de la dimension humaine dans la mutation paysagère récente de certaines zones sahéliennes. A ce titre ils peuvent canaliser les réflexions sur la recherche des facteurs et conditions favorables à la mise à l'échelle du reverdissement.

(C) 2020 International Formulae Group. All rights reserved.

Mots clés : NDVI, Pluviométrie, Anthropisation, Reverdissement, RUE.

\section{Contributing part of population density to the regreening of certain highly anthropized area of the Sahel: the case of Agué and Ibohamane municipalities \\ in Niger}

\section{ABSTRACT}

This study focuses on human dimension in the explanation of the the regreening process observed in certain areas of the Sahel region. For this purpose, the municipalities of Aguié and Ibohamane were chosen 
according to their high population density. The methodology used is based on the application of the correlative analysis coupled with statistical test on the annual rainfall values, population density values and the normalized vegetation index values, over 1980-2018 time series. The results obtained highlight two significant dry and wet sequences. However, the recent wet period, well established from the 1990s does not fully explain the regreening of these areas. Rates of $60 \%$ and $45 \%$ of this change are due to population density respectively in Aguié and Ibohamane case. These results replace the central role of human dimension in the dynamic change of sahelian landscapes and provide the keys that can guide the reflection on the search of factors and conditions favorable of scaling up of regreening.

(C) 2020 International Formulae Group. All rights reserved.

Keywords: NDVI, Rainfall, Anthropization, Regreening, RUE.

\section{INTRODUCTION}

Conventionnellement, le Sahel a toujours été taxé de région où le phénomène de la désertification sévit de façon irréversible (Toudou et al., 2006). Mais contrairement aux affirmation de dégradation continue (Aubréville, 1949) et son irréversibilité (Brown et Wolf, 1985) des nombreux auteurs dont Bégué et al., (2011), Seaquist et al., (2009), Tougiani et al., (2009), Reij et Smaling, (2008), Rinaudo, (2007), Reij et Smaling, (2005) ont montré que dans plusieurs zones du Sahel, un processus de -«végétalisation». Ce phénomène a été amorcé depuis la fin de la sécheresse de 1980 (Olson et al., 2005). Cependant, son évaluation précise n'est pas suffisamment établie. Ozer et al. (2010) suggéraient que la restauration écologique du milieu sahélien correspond à une simple substitution de la savane arbustive par une végétation typiquement saharienne. Ils en donnent l'explication en mettant au premier plan la propension des espèces sahariennes à coloniser les sols sableux sahéliens du fait de leur forte teneur en sable fin. La thèse d'une forte d'une forte influence de la pluviométrie a été mise en avant dans l'explication de ce phénomène (Milich et Weiss ; 2000a, 2000b). Cependant, Hountondji et al. (2006) avaient constaté, au Burkina Faso que l'évolution des rapports de l'efficience d'utilisation des pluies par la couverture végétale est dans l'inertie dans $54 \%$ des stations avec une tendance au déclin dans $44,7 \%$ des cas. C'est dire que le facteur climatique n'explique pas à lui seul la tendance au reverdissement récent observée dans la frange sahélienne.

Abordant dans le même sens, Reij et Smaling (2005) suggèrent qu'il y a d'autres facteurs qui contribuent au reverdissement dans certaines zones sahéliennes. L'action humaine joue un rôle crucial dans cette dynamique de transformation paysagère (Hermann et al., 2005). Les travaux conduits à petite échelle par Baggnian et al. (2014), Larwanou et Saadou (2010), Mortimore (2005), Mahamane (2001), Mazzucato et Niemeijer (2001), Rasmussen (2001) et Raynaut (2001) ont démontré que le reverdissement du Sahel est plus accentué dans les zones de forte anthropisation, donnant ainsi tout son sens à l'expression utilisée par, Seghieri (2017): "plus de gens, plus d'arbres". Il convient alors de mener des études à l'échelle locale pour identifier et comprendre les interactions entre l'environnement en mutation et le facteur humain (Seaquist et al., 2009). Cet article aborde cette question en mettant l'emphase précisément sur la dimension anthropique du reverdissement sahélien. De façon spécifique, il s'agit d'établir le lien entre les dynamiques climatique et paysagère récentes et d'évaluer le rapport entre la densité démographique et la tendance à l'amélioration de la couverture végétale du paysage dans deux communes fortement anthropisées du Sahel. L'intérêt de cette étude réside dans la nécessité de 
comprendre si l'amélioration récente du régime pluviométrique (Ozer et al., 2010 ; Banque Mondiale, 2013 ; Habou et al., 2016), pourrait à elle seule servir de base à l'explication du reverdissement sahélien.

\section{MATERIEL ET METHODES}

\section{Choix des sites d'études}

Deux communes ont été choisies sur la base de leur forte densité démographique et aussi de leur position climatique latitudinale permettant de couvrir le gradient d'aridité Nord-Sud. Il s'agit de la commune rurale
d'Aguié située au Centre-Sud du Niger (Figure 1A) et la commune rurale d'Ibohamane beaucoup plus au Nord à cheval entre la bande saharienne et le Sahel typique dans l'AderDoutchi-Maggia (Figure 1B). Cette dernière fait également office de zone tampon en rempart contre l'avancée du front dunaire saharien vers le Sud.

\section{Caractéristiques des sites d'étude}

Les caractéristiques des sites d'étude sont présentées danns le Tableau 1.
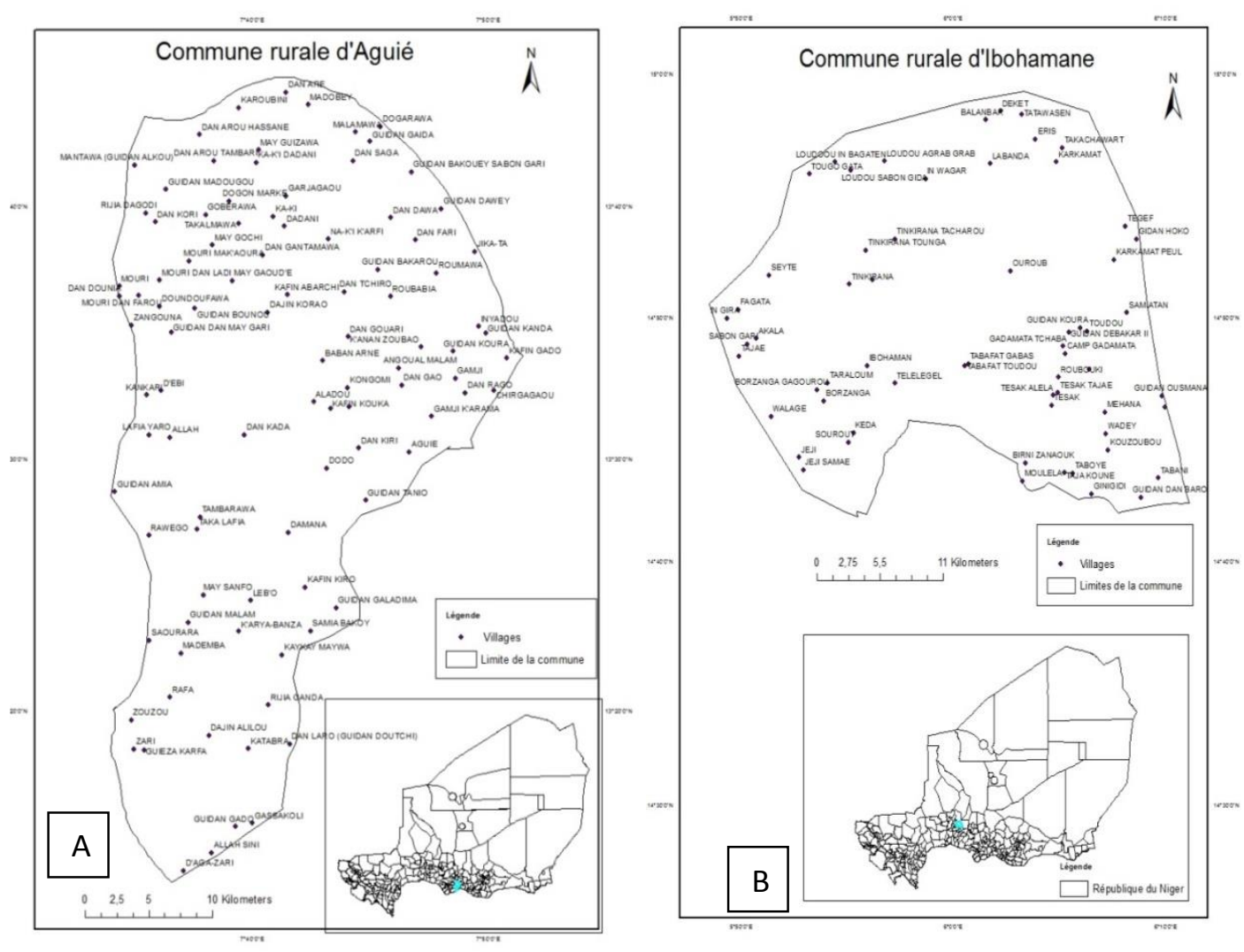

Figure 1 : Localisation de la commune d'Aguié (A) et d'Ibohamane (B) 
Tableau 1 : Caractéristiques des sites d'étude.

\begin{tabular}{|c|c|c|}
\hline & Commnune d'Aguié & Commune d'Ibohamane \\
\hline $\begin{array}{l}\text { Coordonnées } \\
\text { géographiques }\end{array}$ & $\begin{array}{l}07^{\circ} 56 \text { et } 07^{\circ} 85 \text { de Longitude Est et } \\
13^{\circ} 23 \text { et } 13^{\circ} 74 \text { de latitude Nord } \\
\text { (Institut National de la Statistique, } \\
\text { 2015) }\end{array}$ & $\begin{array}{l}5^{\circ} 50^{\prime} \text { et } 6^{\circ} 00^{\prime} \text { de longitude Est, } 14^{\circ} 40^{\prime} \text { et } \\
14^{\circ} 50^{\prime} \text { de latitude Nord (Institut National de la } \\
\text { Statistique, 2015) }\end{array}$ \\
\hline $\begin{array}{l}\text { Densité } \\
\text { démographiques } \\
\text { (données du RGPH, } \\
\text { 2011) }\end{array}$ & $\begin{array}{l}137,64 \text { habitants } / \mathrm{km}^{2} \quad \text { (Institut } \\
\text { National de la Statistique, 2015) }\end{array}$ & $\begin{array}{l}100 \text { habitants } / \mathrm{km}^{2} \text { (Institut National de la } \\
\text { Statistique, 2015) }\end{array}$ \\
\hline Superficie & $\begin{array}{l}1110 \mathrm{~km}^{2} \text { (Institut National de la } \\
\text { Statistique, 2015) }\end{array}$ & $\begin{array}{l}1875 \mathrm{~km}^{2} \text { (Institut National de la Statistique, } \\
2015)\end{array}$ \\
\hline Climat & $\begin{array}{l}\text { Climat semi-aride steppique d'après } \\
\text { la classification de Koppen-Geiger }\end{array}$ & $\begin{array}{l}\text { Climat aride steppique d'après la classification } \\
\text { de Koppen-Geiger }\end{array}$ \\
\hline Relief & $\begin{array}{l}\text { Vaste plateau d'ondulations } \\
\text { sableuses, ponctués des édifices } \\
\text { dunaires (Ambouta et al., 1998). }\end{array}$ & $\begin{array}{l}\text { Plateaux gréseux assez bien contrasté entaillé } \\
\text { par des vastes vallées fertiles (Gavaud, 1968) }\end{array}$ \\
\hline Types de sols & $\begin{array}{l}\text { Sols ferrugineux tropicaux non ou } \\
\text { peu lessivés dunaires à texture } \\
\text { sableuse, sols ferrugineux tropicaux } \\
\text { lessivés et sols hydromorphes des } \\
\text { vallées (Ambouta et al., 1998). }\end{array}$ & $\begin{array}{l}\text { Sols bruns rouges subarides dunaires, } \\
\text { Régosols des glacis et plateaux encroûtés et } \\
\text { sols hydromorphes et vertisols des vallées } \\
\text { (ORSTOM, 1964) }\end{array}$ \\
\hline Végétation & $\begin{array}{l}\text { Faciès à combrétacées (Morou, } \\
\text { 2016) }\end{array}$ & Faciès à mimosacées (Garba, 2017) \\
\hline
\end{tabular}

\section{Données}

Trois types des données ont été utilisés dans le cadre de cette étude. Il s'agit des cumuls pluviométriques annuels et les valeurs mensuelles de l'Indice Normalisé de Végétation (NDVI) et les valeurs annuelles de la population exprimée en nombre d'habitants dans les deux communes.

Les données du NDVI et les cumuls pluviométriques ont été générées par télédétection sur les images satellitaires de type panchromatique respectivement extension TIFF et extension BILL à l'aide du logiciel ArcGIS 10.3. Ces images ont été acquises auprès du centre régional AGRHYMET. Elles couvrent la série 1981 à 2018. Les données démographiques sont extraites du Répertoire National des Localités (RENALOC) version 2014 (Institut National de la Statistique, 2014).
Analyse des données

Caractérisation des tendances
pluviométriques des communes étudiées
L'extraction des valeurs de la pluviométrie surfacique et celles du NDVI a été faite à l'aide du logiciel ArcGIS 10.3 à partir des tables attributaires des couches raster sur la série 1980 à 2018. L'utilisation du module zonal statistique du logiciel a permis de générer les hauteurs des pluies mensuelles. En ce qui concerne les valeurs maximales mensuelles du NDVI, elles ont été extraites par le moyen du module local cell statistique du logiciel.

La caractérisation des tendances pluviométriques repose sur l'hypothèse selon laquelle la valeur moyenne des pluies générées par le logiciel ArcGIS 10.3 à partir de tous les pixels pour une année donnée, est représentative de la hauteur de pluie obtenue au cours de l'année correspondante. Elle exclut à ce titre les variabilités spatiales qui constituent 
tout de même une des caractéristiques importante de la pluviométrie en zone sahélienne. L'analyse a consisté à analyser les variabilités interannuelles des pluies sur la série chronologique et à établir les séquences sèches et humides qui ont émaillé les deux communes. A cet effet, la formule de Lamb (1982) a été utilisée pour calculer les indices standardisés des pluies annuelles de la série 1980 à 2018. L'équation de Lamb est donnée par le bilan :

$I S P=\frac{X-\mu}{\sigma} \quad$ (Equation 1)

où $X$ désigne la pluviométrie de l'année i, $\mu$ désigne la valeur moyenne de la pluviométrie de la série considérée et $\sigma$ désigne l'écart type.

L'équation 1 a permis d'apprécier les anomalies pluviométriques des deux communes.

La décomposition d'une série cherche à identifier soit une tendance, une périodicité, une autocorrélation ou des aléas. La tendance décline l'évolution globale monotone de la série, bien délimitée par des ruptures. Elle peut être progressive, régressive ou stationnaire. La périodicité traduit une oscillation régulière autour d'une tendance. L'autocorrélation donne la mesure de l'interdépendance entre les valeurs consécutives, ce qui donne une appréciation de l'effet mémoire sur la série. Les aléas sont les faits d'événements exceptionnels.

La décomposition de cette série a été réalisée à l'aide du logiciel Khronostat 1.01 , mis au point par l'institut hydro-science Montpellier IRD (2002). Elle a consisté dans un premier temps à vérifier l'interdépendance des pluies des années successives sur la fonction d'autocorrélation. L'hypothèse $\mathrm{H} 0$ permet de conclure à la non stationnarité de la série. D'où l'intérêt de rechercher les points de rupture sur la série. La mise en évidence des points de rupture a été faite au moyen du test de Pettit (1979). C'est une approche non paramétrique déduite du test de MannWithney, particulièrement sensible au changement des moyennes de la série. Lorsque l'hypothèse H0 est acceptée, les variables suivent une distribution ayant une moyenne sensiblement constante sur la série. Dans ce cas on admet qu'il y'a une seule tendance. Si en revanche, $\mathrm{H} 0$ est rejetée, on procède à la détermination du point de rupture. Une rupture est un changement dans la loi de probabilité de la série chronologique à un instant donné. Deux autres tests ont été appliqués à la série étudiée pour bien démarquer les tendances. Le premier, le test de vraisemblance des moyennes de Buishand (1982), de nature bayésienne, permet en même temps de vérifier la normalité de la distribution. La validité du test est matérialisée par les courbes de contrôle représentées par l'Ellipse du Bois. Trois niveaux de confiance : $90 \%, 95 \%$ et $99 \%$ permettent de visualiser le niveau significatif d'homogénéité de la distribution de la série. Le deuxième, la segmentation de Hubert (1989), a permis de déterminer des segments de périodes dont les moyennes et les écart-types sont significativement différents à l'épreuve du test de Scheffé au seuil de $1 \%$. La procédure de Lee et Heghinian précise l'amplitude du changement du régime pluviométrique sur une série temporelle (Buishand, 1982).

\section{Analyse des liens entre le reverdissement et le régime pluviométrique}

La détermination des liens entre le NDVI et la pluviométrie a été obtenue à travers le calcul de l'indice RUE (Rain Use Efficiency) qui reflète l'efficacité d'utilisation des pluies par le couvert végétal d'un paysage. Cet indice est fréquemment utilisé dans la littérature récente (Hermann et al., 2005 ; Prince et al., 1998). Son usage est particulièrement pertinent dans les régions arides et semi-arides où la pluviométrie est considérée comme étant le facteur limitant de la production végétale (Le Houerou, 1984). Le RUE s'obtient par la formule :

$R U E=\frac{N D V I_{\max }}{\text { Pluviométrie }} * 100$ (Equation 2).
Dans l'équation 2, le $N D V I_{\max }$ désigne la valeur mensuelle maximale du NDVI au cours de la période végétative (juin, juillet et août) de l'année considérée et la Pluviométrie correspond au cumul pluviométrique correspondant. Le $R U E$ est une estimation de la biomasse aérienne nette obtenue à la suite d'une saison pluviométrique (Dardel et al., 2014). Sa diminution est synonyme d'une dégradation du couvert. L'augmentation de cet indice exprime en revanche l'influence de la pluviométrie sur l'amélioration de la couverture végétale. Dans cette analyse, toute valeur négative ou nulle du RUE permettra d'exclure la contribution 
climatique à l'amélioration de la couverture végétale et donc d'en conclure à une origine anthropique. L'intérêt de considérer la période de saison végétative permet de réduire l'influence des sources d'erreur et d'incertitudes liées à l'usage de cet indice (Fensholt et al., 2016).

Ensuite, l'appréciation des courbes d'évolution a permis de mettre en parallèle les deux paramètres NDVI_max et RUE. Les plages temporelles au cours desquelles ces deux paramètres divergent confirment l'influence anthropique sur la tendance au reverdissement. Dans cette perspective, des tests de corrélation de Pearson au seuil de 5\% ont été appliqués sur les deux paramètres pour démontrer le niveau de leur interdépendance.

\section{Quantification de la part anthropogénique au reverdissement}

Pour mieux conforter les résultats trouvés par la méthode RUE, il est apparu nécessaire de recourir à la méthode dite des «résidus» proposée par Van et Geerken (2004). Cette méthode consiste à établir dans un premier temps, une régression linéaire entre les valeurs du NDVI et la pluviométrie. L'équation de la régression linéaire est obtenue par la formule :

$$
Y=a X+b+\varepsilon(\text { Equation 3). }
$$

Sur l'équation $3, Y$ est la valeur théorique du NDVI sur le modèle, $X$ est la pluviométrie correspondante, $a$ est le coefficient de corrélation de Pearson des deux paramètres, b l'ordonnée à l'origine de la courbe de régression et $\varepsilon$ est la marge d'erreur. En prenant en compte cette équation, on a calculé des valeurs théoriques de NDVI de la série chronologique 1980-2018.

Les deux paramètres NDVI_max et le RUE sont liés par les équations des droites de régression linéaires suivantes : pour le cas de la commune d'Aguié : $Y=0,34 X+0,7$ (équation 4) et pour le cas de commune d'Ibohamane: $Y=0,83 X+0,08$ (équation 5).

Les équations 4 et 5 ont permis de calculer les valeurs théoriques du paramètre NDVI_max dans les deux cas. Les valeurs des résidus sont obtenues en faisant la différence entre les valeurs mesurées et les valeurs théoriques. Ces résidus constituent une estimation quantitative de la part anthropogénique du reverdissement.
Dans cette étude les paramètres statistiques mis en cause sont notamment les valeurs maximales, minimales, moyenne, écart-type et coefficient de variation. Le coefficient de variation $\mathrm{CV}$ est défini selon la formule :

$$
C V=\frac{\text { Ecart-type }}{\text { Moyenne }} * 100 \text { (Equation 6). }
$$

Les paramètres statistiques globaux constituent des référentiels dans l'estimation de la part anthropogénique sur le reverdissement.

\section{Evaluation des liens entre dynamique anthropique et tendance au reverdissement}

Le paramètre densité démographique a été retenu pour évaluer les liens entre la dynamique anthropique et la tendance au reverdissement. Il a fallu pour cela déterminer la dynamique de la population des deux sites sur la série chronologique considérée. Cela a été fait en utilisant la formule préconisée par l'Institut de la Statistique National du Niger (2012) qui est de la forme :

$P_{n+1}=P_{n}(1+i)^{n}$ (Equation 7).

Les variables $P_{n+1}$ et $P_{n}$ désignent respectivement les valeurs annuelles de population au cours de l'année $n+1$ et $n$. La variable $i$ représente le taux de croissance intercensitaire. Ce taux est constant à $3,7 \%$ au niveau de la région de Maradi et a été considéré pour la commune d'Aguié (Institut National de la Statistique, 2012). Par contre au niveau de la région de Tahoua ce taux varie suivant trois plages temporelles : 2,6\% de 1977 à 1988, $3,2 \%$ de 1989 à 2001 et 4,7\% entre 2002 à 2012 (Institut National de la Statistique, 2015). C'est pourquoi on a appliqué les différents taux de croissance intercensitaire par décennie correspondante pour calculer les nombres annuels d'habitants sur la série chronologique 1980-2018 dans le cas de la commune d'Ibohamane. Les valeurs de références utilisées dans les calculs sont celles issues des Recensements Généraux de la Population et de l'Habitat (RGPH) réalisés successivement en 1977, 1988, 2001 et 2012. Face à la non disponibilité des données à l'échelle de la commune pour les deux premiers recensement et aussi des changements ultérieurs opérés sur la carte administrative des collectivités territoriales en république du Niger, il est apparu nécessaire de prendre en compte l'échelle départementale en gardant l'hypothèse qu'à cette échelle les densités sont 
suffisamment représentatives de celles des communes. En fait l'échelle départementale représente le plus bas niveau de désagrégation des résultats de RGPH 1977 et RGPH 1988.

Les données démographiques calculées ont été croisées à celles du paramètre NDVI_max pour évaluer le niveau de corrélation entre la densité démographique et la tendance au reverdissement observée sur les deux paysages concernés par cette étude. Lorsque le coefficient de corrélation $\mathrm{R}$ est significativement positif, on pourra conclure à une forte influence anthropique sur l'amélioration de la couverture végétale du paysage.

\section{RESULTATS}

\section{Importance de la densité démographique dans les deux communes}

Dans le cadre de cette étude, l'anthropisation est exprimée par la densité démographique sur le paysage. Le Tableau 2 récapitule les valeurs des paramètres statistiques globaux des densités démographiques calculées sur la série 1980 à 2018 grâce à l'équation 7. Il ressort de l'examen de ce tableau que sur quatre des cinq paramètres statistiques, la densité démographique est notablement plus élevée à Aguié par rapport à Ibohamane, avec une moyenne globale de 85,42 à Aguié contre 43,99 à Ibohamane. Le coefficient de variation de la densité démographique au niveau d'Ibohamane est en revanche plus élevé que celui d'Aguié $(37,93 \%$ à Ibohamane contre $31,05 \%$ à Aguié) suggérant que la distribution de la densité démographique n'est pas homogène dans le cas de cette commune, sur la série chronologique considérée.

\section{Caractéristiques des tendances pluviométriques dans les deux communes}

L'indice standardisé des pluies (ISP) de Lamb traduit la variabilité interannuelle des pluies sur la série. L'examen des Figures 2 et 3 des anomalies pluviométriques permet de déceler quatre périodes de déficits pluviométriques qui alternent avec des périodes notablement plus humides. Cette fluctuation est beaucoup plus prononcée au niveau d'Aguié. On observe un relatif fléchissement à tendance sèche dans le cas d'Ibohamane.

\section{Niveau d'interdépendance entre les hauteurs de pluies annuelles successives}

Les autocorrélogrammes des Figures 4 et 5 montrent que dans les deux communes, le niveau d'interdépendance entre les hauteurs des pluies successives augmente au cours de la première décennie. Puis on observe une cassure significative au seuil de confiance de $90 \%$. La fonction d'autocorrélation évolue progressivement avec une allure en dents de scie dans le cas d'Aguié et une quasimonotonie dans le cas d'Ibohamane. Dans le cas d'Aguié, le résultat du test de corrélation sur le rang indique que l'hypothèse nulle est rejetée au seuil de 90 et $95 \%$ et la valeur de la variable de calcul au rang est de 2,55. Dans le cas d'Ibohamane, le test de corrélation au rang accepte l'hypothèse nulle avec une valeur beaucoup plus faible de 1,44. Au seuil de confiance de $90 \%$ la distribution temporelle des pluies au niveau d'Ibohamane est plus ou moins homogène.

Domaine de validité de l'amplitude de changement du régime pluviométrique

Le test de Buishand est applicable uniquement à la série d'Aguié parce que la distribution des données pluviométriques dans ce cas suit une loi normale. A l'épreuve de ce test, l'hypothèse nulle est rejetée au seuil de 90\% comme le montre les trois ellipses du Bois de la Figure 6 qui indiquent deux points de rupture en 1993 et en 1997. Les résultats de la procédure de Lee et Heghinian représentés dans la Figure 7 affichent que le changement du régime pluviométrique est positif avec une amplitude de 0,014 dans le cas de la commune d'Aguié.

\section{Détection de point de rupture par le test de Pettit}

L'application des tests de Pettit sur les deux séries donne les résultats présentés par les Figures 8 et 9 . Sur la situation d'Aguié on peut déceler deux tendances nettement démarquées par la valeur critique $3,45.10^{-3}$ en 1997 du test sur la fonction $U$ de probabilité de dépassement. La première tendance de type régressive correspond à la période de 1980 à 1997. La deuxième tendance de type progressive couvre la période 1998-2018. 
Dans le cas d'Ibohamane, la rupture n'est pas statistiquement significative, mais on observe un point de rupture en 1987. Selon l'allure de la courbe d'évolution de la fonction $\mathrm{U}$ du test de Pettit, trois tendances peuvent être définies. Une première tendance régressive beaucoup plus tranchée sur la période de 1980 à 1987. La deuxième phase va de 1987 à 1997. Cette phase est à tendance stationnaire caractérisée par une forte fluctuation interannuelle. Cependant, à partir de 1997 on observe une tendance progressive caractérisée par une forte fluctuation interannuelle des pluies.

\section{Identification des séquences pluviométriques}

Le Tableau 3 montre que dans les deux communes, la segmentation de Hubert met en évidence deux séquence pluviométriques hautement significatives au test de Scheffé. Une séquence humide assez nette qui commence à partir de 1993 et 1987 respectivement au niveau de la commune d'Aguié et celle d'Ibohamane. Cette séquence succède à une période sèche. De plus, dans les deux cas la variabilité est beaucoup plus importante sur la séquence sèche comparée à la dernière période. Sur le dernier segment, la fluctuation est nettement plus importante à Ibohamane $(\mathrm{CV}=17,32 \%$ contre $14,78 \%$ à Aguié).

\section{Rapport entre l'évolution climatique et l'intensité de la couverture végétale}

Les Figures 10 et 11 représentent les courbes d'évolution du NDVI_max et du RUE. Ces deux courbes montrent une allure en dent de scie caractéristique des fluctuations pluviométriques interannuelles de la série considérée. Ainsi, on observe qu'en 19802002, 2004-2006, 2009-2014 les valeurs du RUE déclinent alors que celles du NDVI augmentent. Cette évolution antagoniste est beaucoup plus prononcée au niveau de la commune d'Ibohamane où on enregistre une nette diminution du RUE et concomitamment une augmentation du NDVI ponctuée de pics en particulier sur la plage 1980-2005.

\section{Niveau de corrélation entre le NDVI et le RUE}

Le niveau de corrélation entre le NDVI et le RUE est évaluée par une analyse corrélative entre les trois paramètres, pluviosité, NDVI_max et RUE. Le Tableau 4 donne les valeurs de coefficients de corrélation $\mathrm{R}$ de Pearson entre les trois paramètres par commune. Les résultats de cette analyse confirment que la corrélation entre la pluviométrie et le NDVI_max est très faible dans les deux cas $(\mathrm{R}=0,35$ pour le cas d'Aguié et $\mathrm{R}=0,11$ pour le cas d'Ibohamane). Par contre, la pluviométrie exprime une corrélation négativement significative avec le RUE ( $R=-$ 0,52 avec $\mathrm{p}$-value $=0,016$ pour le cas d'Aguié et $\mathrm{R}=-0,45$ avec $\mathrm{p}$-value $=0,040$ pour le cas d'Ibohamane). Les deux communes s'opposent nettement au test de corrélation entre la pluviométrie annuelle et les valeurs de RUE. De plus, au niveau de la commune d'Aguié, l'hypothèse de l'indépendance des deux paramètres NDVI_max et RUE est confirmée. $\mathrm{Au}$ niveau de la commune d'Ibohamane, le NDVI_max présente un niveau de corrélation très significatif avec le RUE ( $R=0,83$ et $p$-value $<0,0001)$.

\section{Quantification de la part anthropogénique sur le reverdissement}

Le Tableau 5 indique que la part anthropogénique maximale est plus importante à Aguié comparativement à Ibohamane. Elle est représentée par des valeurs de NDVI variant de 0,04 à 0,24 au niveau d'Aguié contre une fourchette de 0,01 à 0,18 pour le cas d'Ibohamane. La Figure 12 précise le niveau de cette contribution de la densité démographique à $60 \%$ et $45 \%$ du reverdissement respectivement à Aguié et Ibohamane.

\section{Lien entre niveau d'anthropisation du paysage et tendance au reverdissement}

Le Tableau 6 présente le niveau de corrélation et les valeurs de p-values correspondantes entre la densité démographique et l'indice NDVI. En considérant les valeurs de p-values la corrélation n'est pas statistiquement significative entre les deux paramètres. En revanche les valeurs de $\mathrm{R}$ indique que le lien entre les deux paramètres est considérable (supérieur à 50\%) avec 0,63 à Aguié et 0,51 à Ibohamane. 
Tableau 2 : Paramètres statistiques globaux des densités démographiques sur la série 1977-2018 des deux communes.

\begin{tabular}{lcc}
\hline Localité & Aguié & Ibohamane \\
\hline Maximum $\left(\mathrm{Hbts} / \mathrm{km}^{2}\right)$ & 140,69 & 83,83 \\
Moyenne $\left(\mathrm{Hbts} / \mathrm{km}^{2}\right)$ & 85,42 & 43,99 \\
Minimum $\left(\mathrm{Hbts} / \mathrm{km}^{2}\right)$ & 44,68 & 24,06 \\
Ecart-type & 26,52 & 16,68 \\
Coefficient de variation $(\%)$ & 31,05 & 37,93 \\
\hline Légende : Hbts : habitants. & &
\end{tabular}

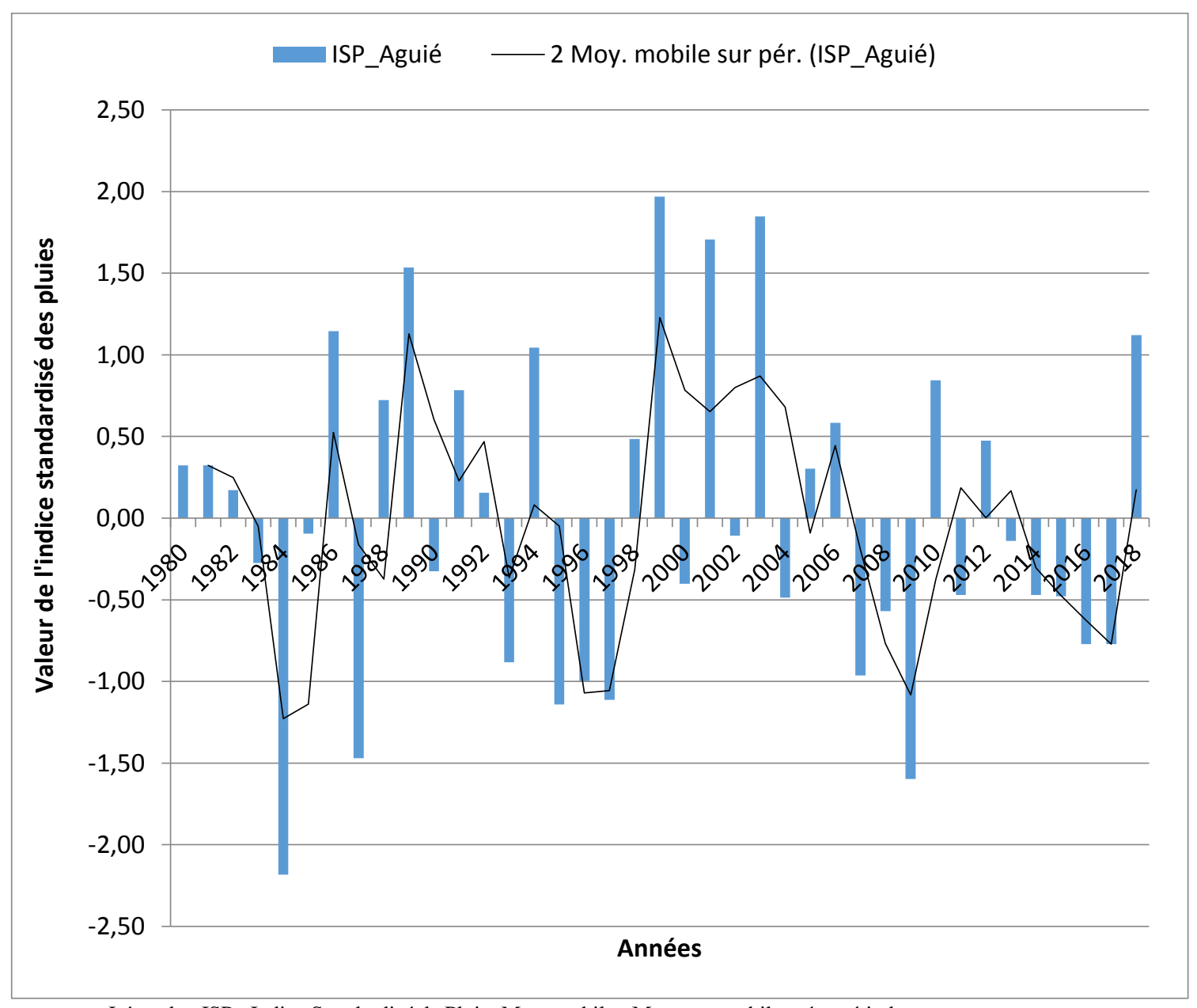

Légende : ISP : Indice Standardisé de Pluie, Moy. mobile : Moyenne mobile, pér : période

Figure 2 : Evolution de l'indice standardisé des pluies (ISP) à Aguié. 


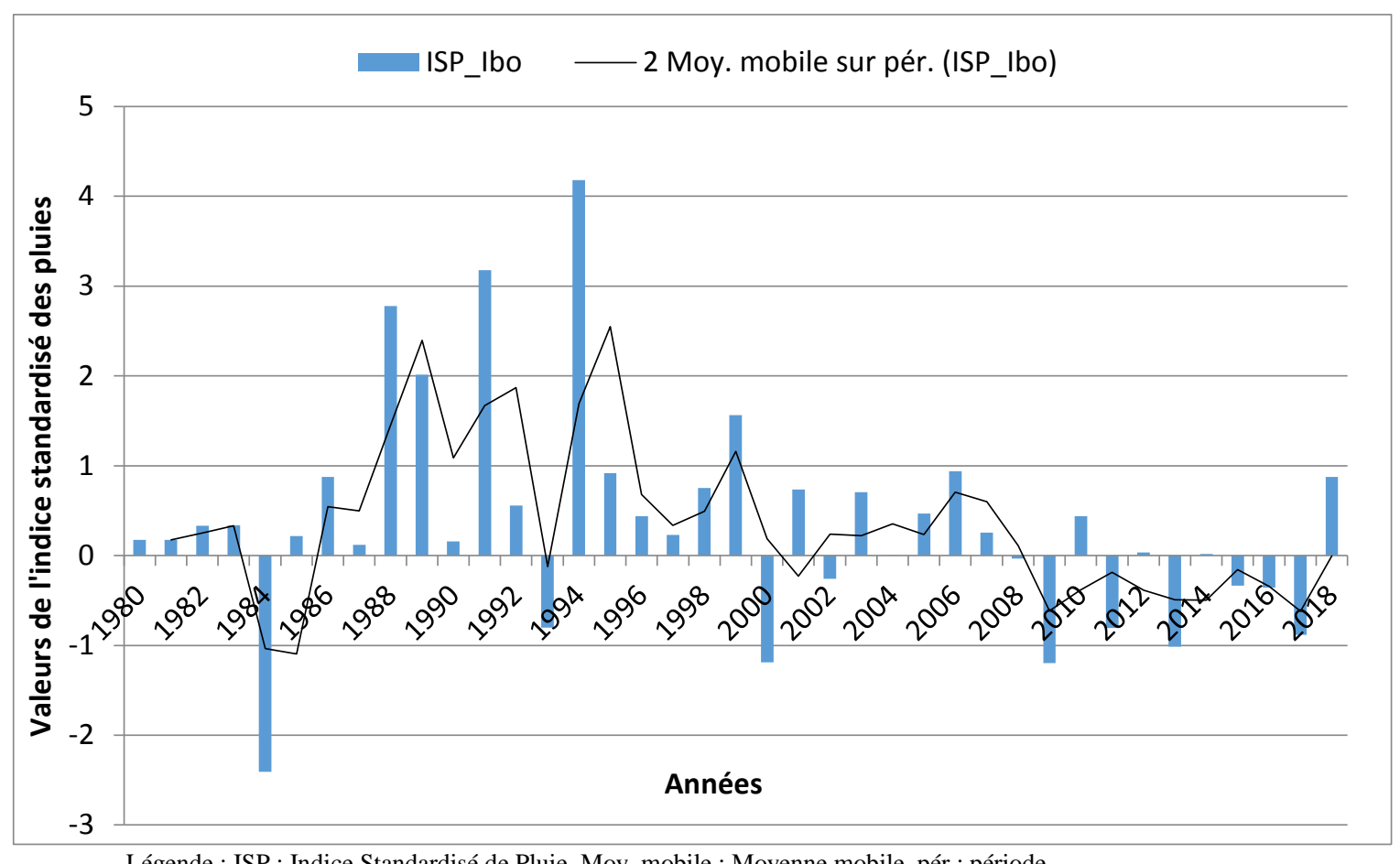

Légende : ISP : Indice Standardisé de Pluie, Moy. mobile : Moyenne mobile, pér : période

Figure 3 : Evolution de l'indice standardisé des pluies (ISP) à Ibohamane.

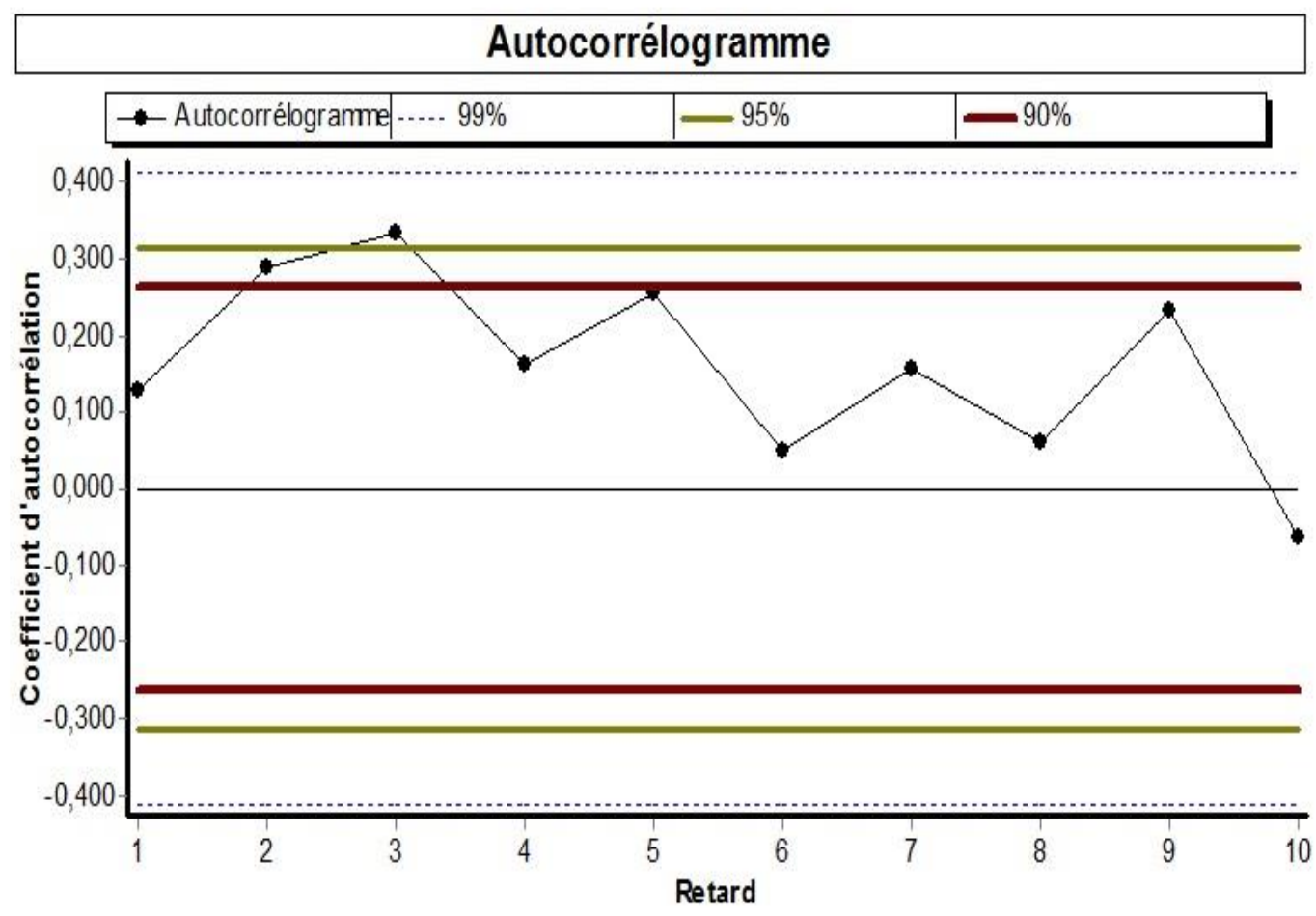

Figure 4 : Autocorrélogramme des pluies à Aguié. 


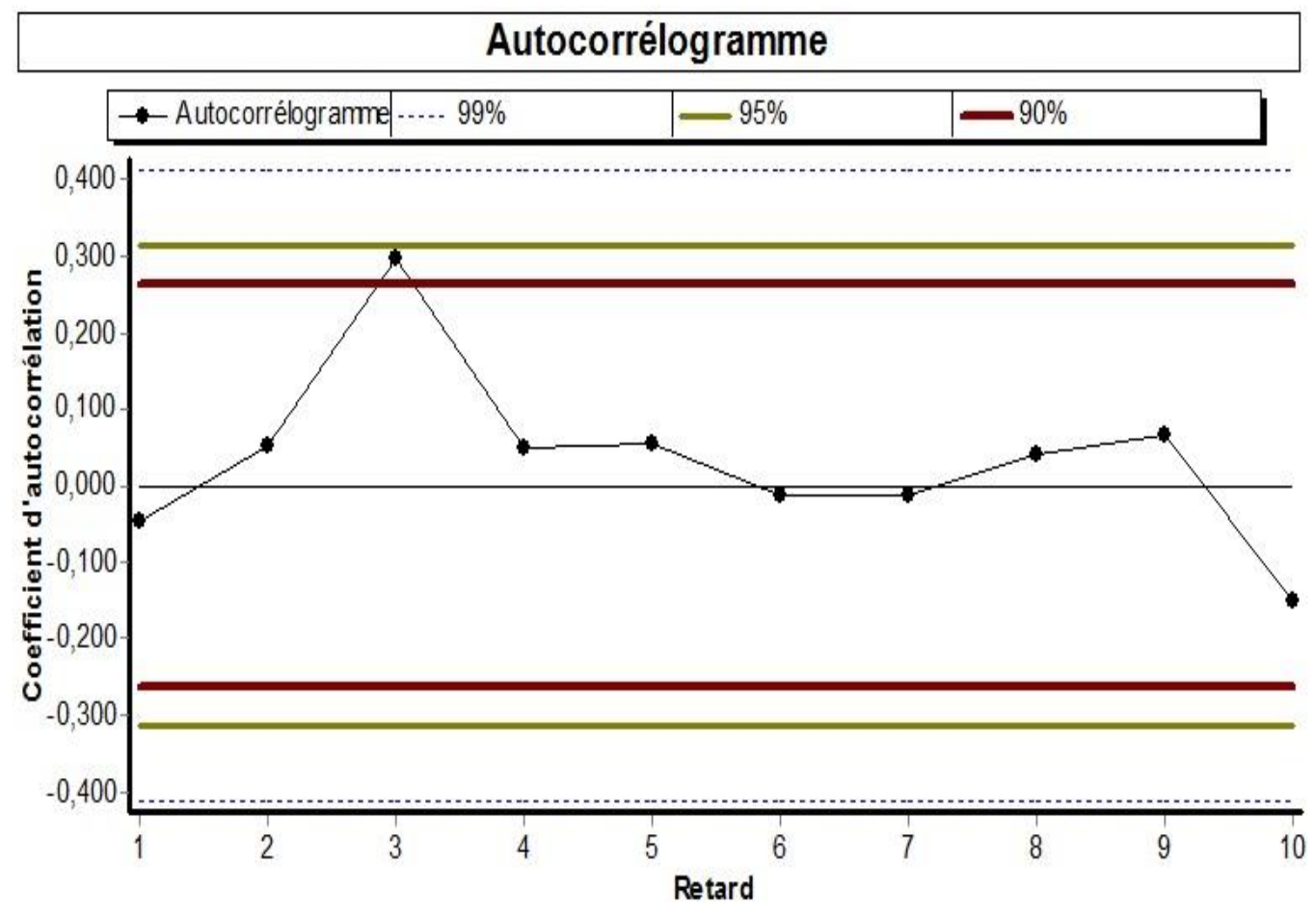

Figure 5 : Autocorrélogramme des pluies à Ibohamane.

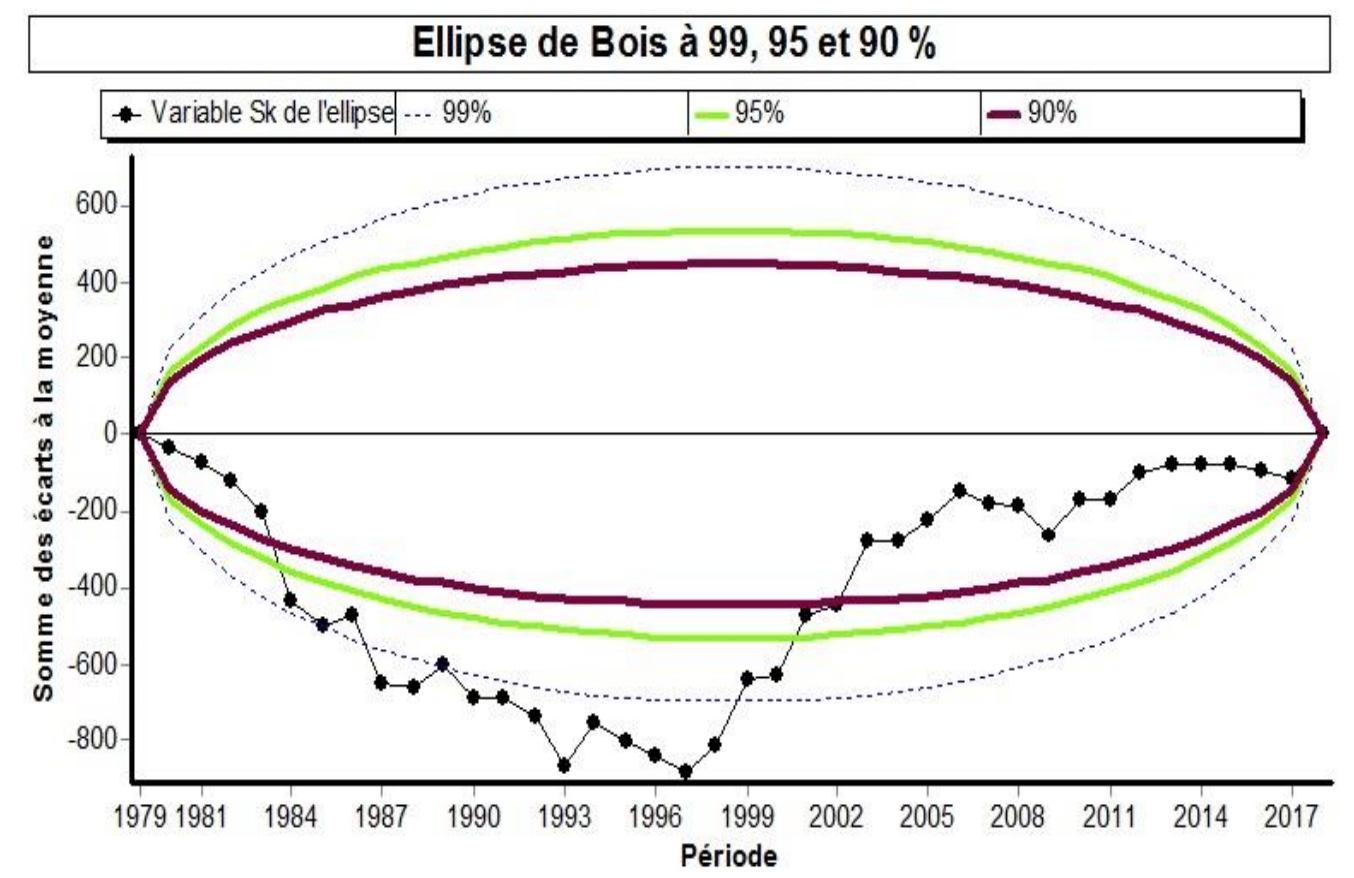

Figure 6 : Distribution des sommes des écart-types cas d'Aguié. 


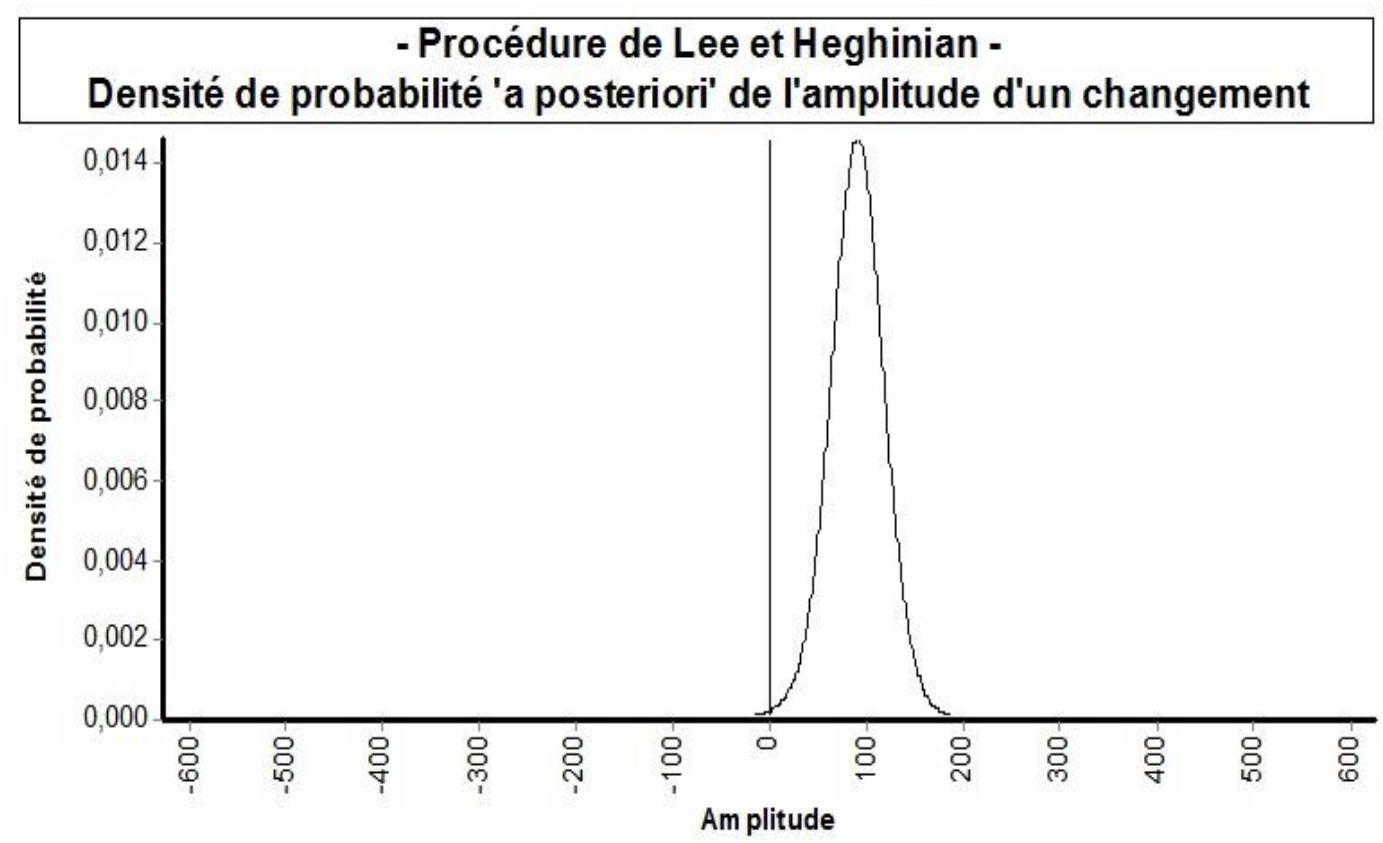

Figure 7 : Distribution des densités de probabilité de l'amplitude de changement Aguié.

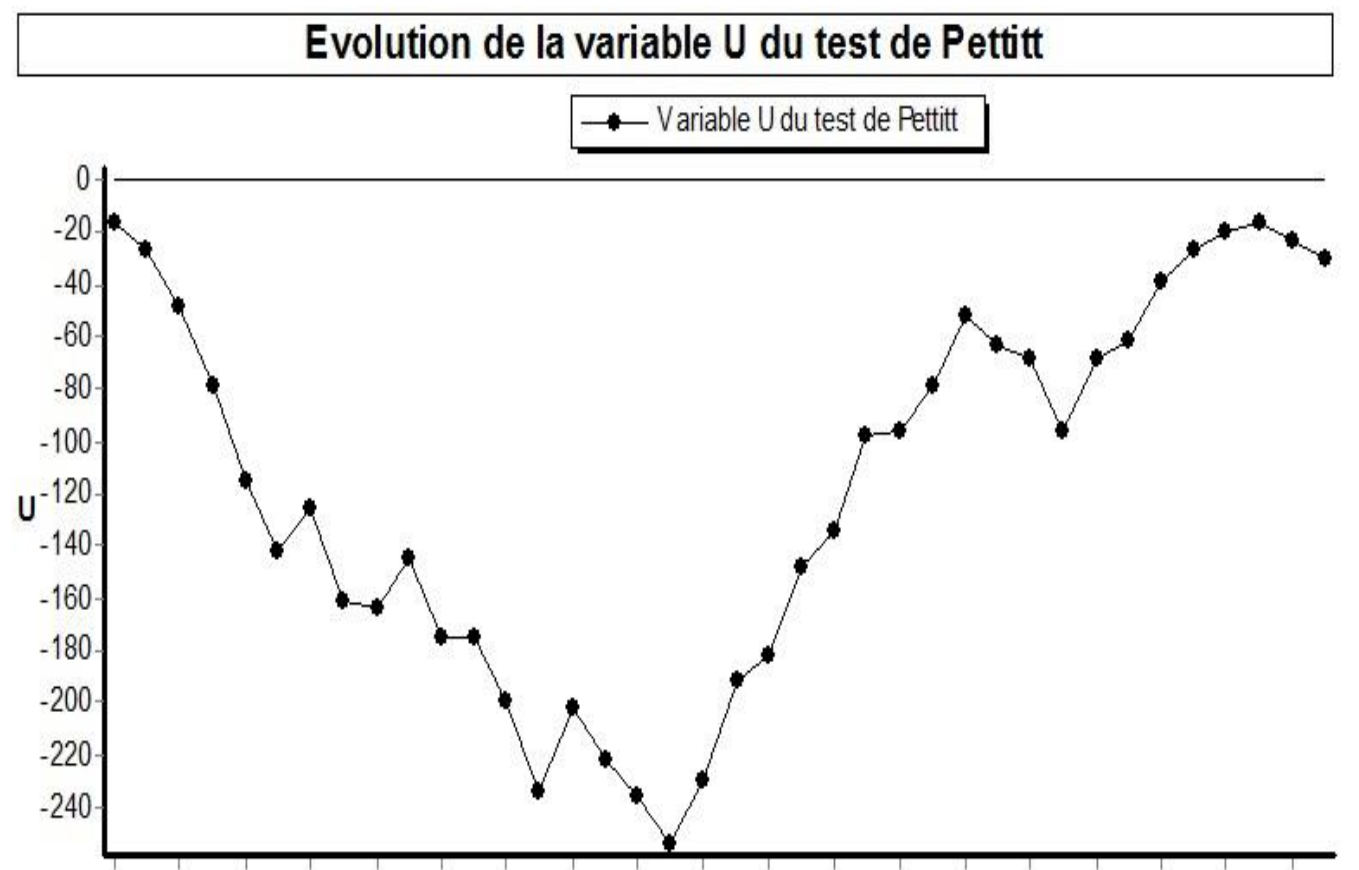

1980198219841986198819901992199419961998200020022004200620082010201220142016

Période

Figure 8 : Evolution de la variable U du test de Pettit à Aguié. 


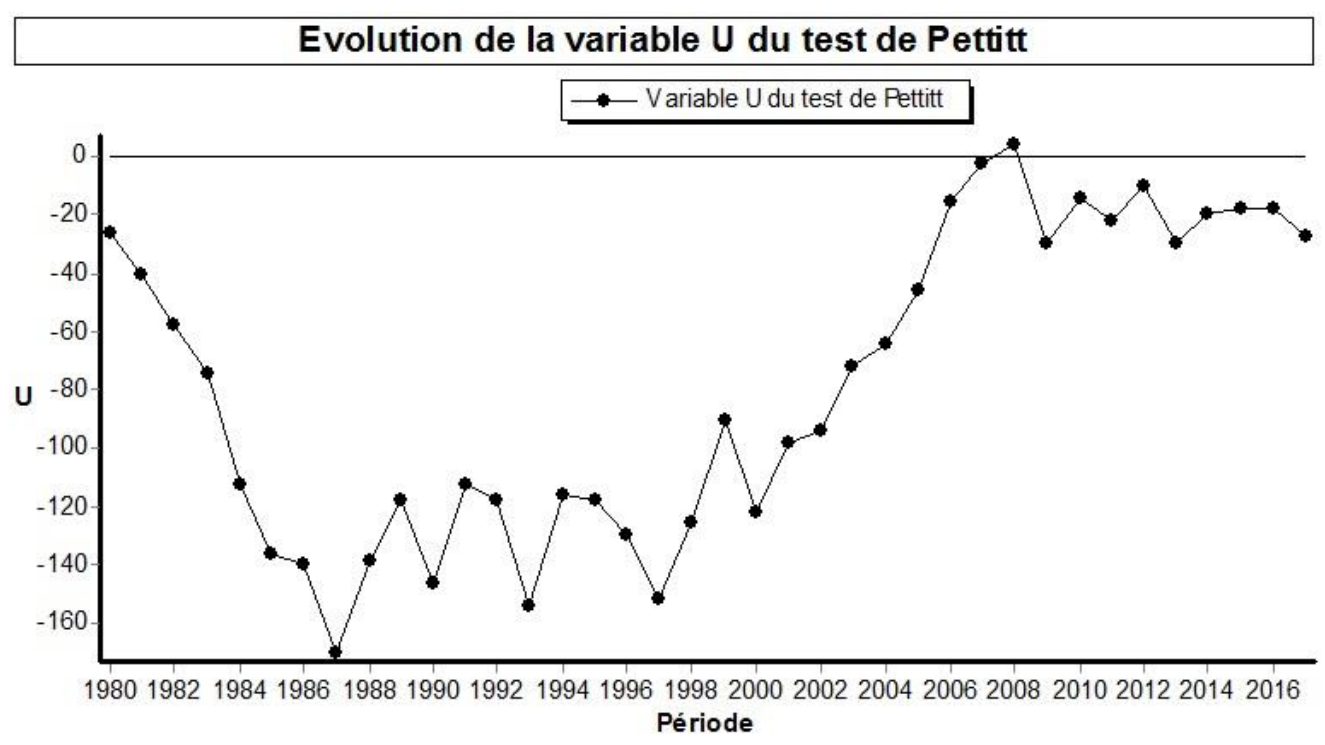

Figure 9 : Evolution de la variable U du test de Pettit à Ibohamane.

Tableau 3 : Résultats de la segmentation de Hubert sur la série chronologique 1980-2018.

\begin{tabular}{llllllll}
\hline Localité & \multicolumn{3}{c}{ Aguié } & \multicolumn{5}{c}{ Ibohamane } \\
\hline Période & Moy $(\mathbf{m m})$ & E-T & CV $(\boldsymbol{\%})$ & Période & Moy $(\mathbf{m m})$ & E-T & CV (\%) \\
\hline $1980-1993$ & 388,14 & 78,17 & 20,14 & $1980-1987$ & 278,89 & 55,55 & 19,92 \\
$1994-2018$ & 485,19 & 71,71 & 14,78 & $1988-2018$ & 359,14 & 62,21 & 17,32 \\
\hline \multicolumn{2}{c}{ Moy : moyenne du segment, E-T : Ecart-type de segment et CV : Coefficient de Variation. }
\end{tabular}

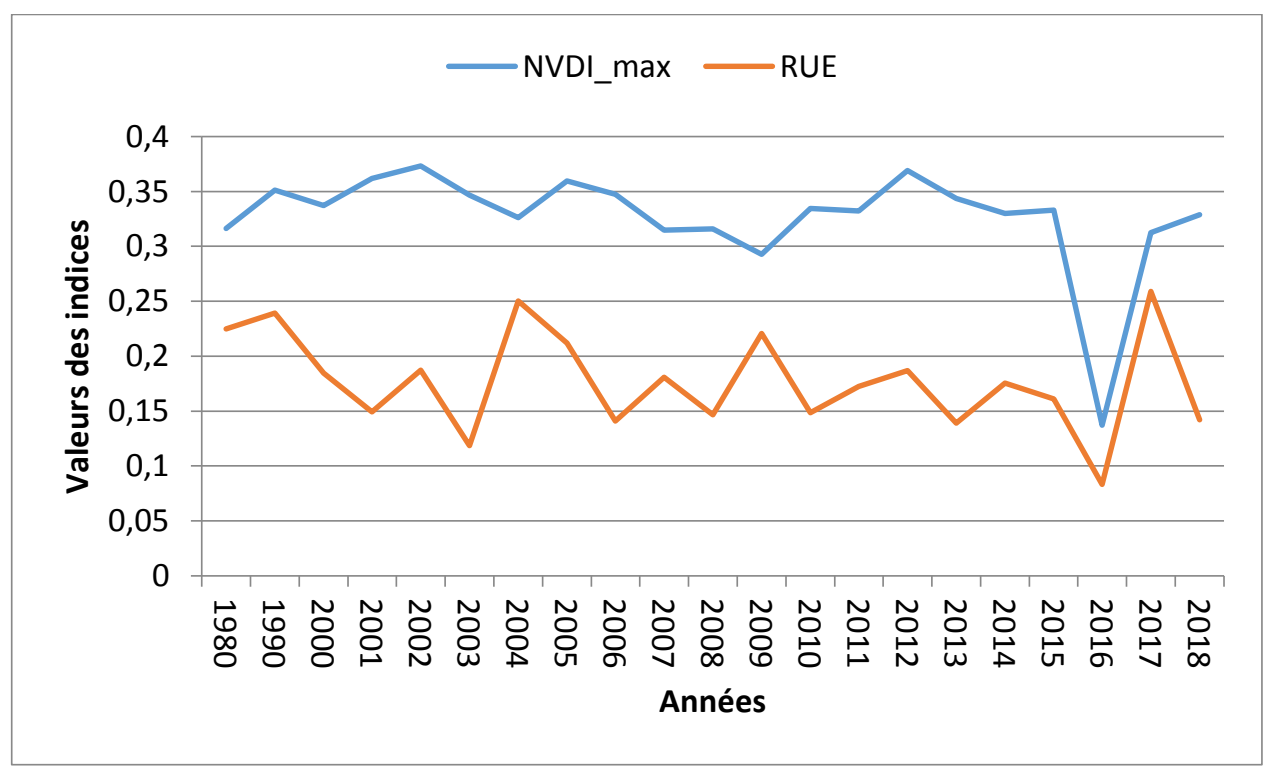

Légende : NDVI_max : Indice Normalisé de Végétation maximal, RUE : Indice d'Efficacité d'Utilisation de Pluie

Figure 10 : Evolution des indices NDVI_max et RUE dans la commune d'Aguié. 


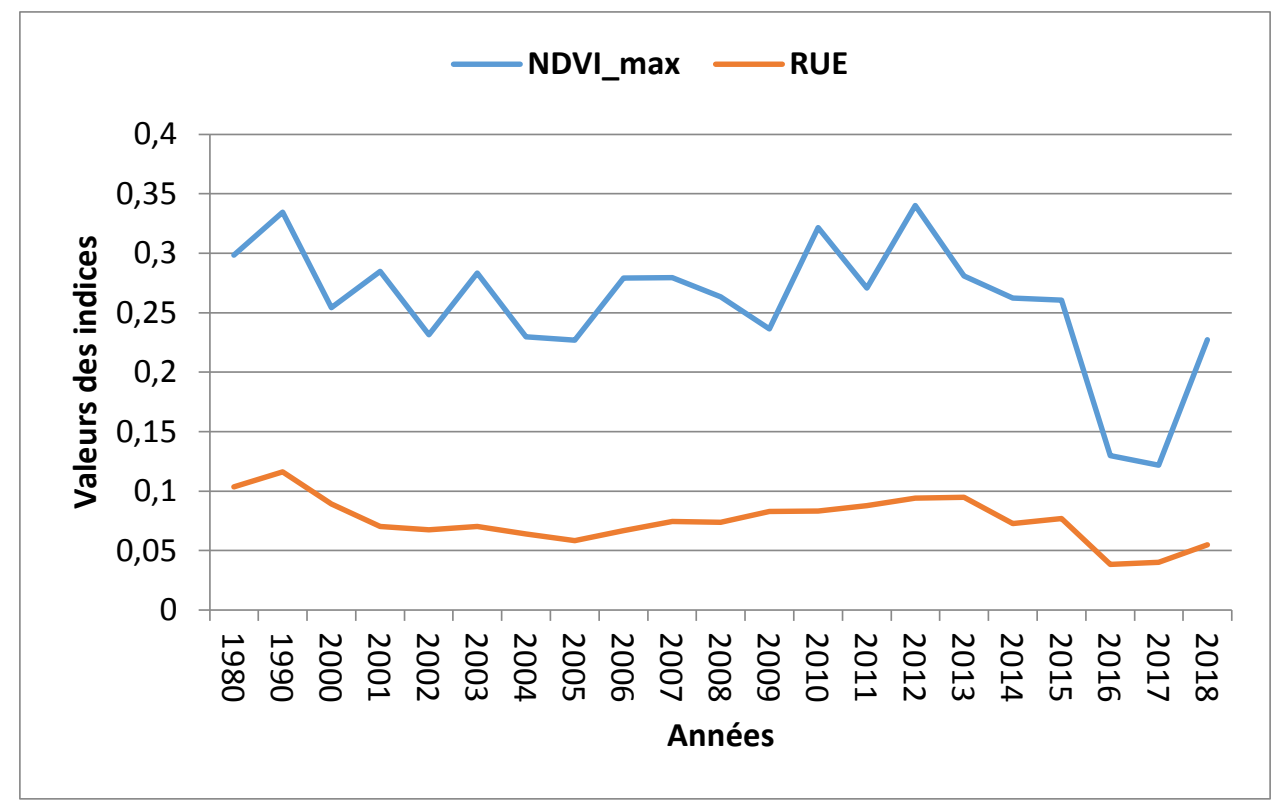

Légende : NDVI_max : Indice Normalisé de Végétation maximal, RUE : Indice d'Efficacité d'Utilisation de Pluie.

Figure 11 : Evolution des indices NDVI_max et RUE dans la commune d'Ibohamane.

Tableau 4 : Niveau de corrélation de Pearson entre Pluie NDVI_max et RUE.

\begin{tabular}{lcccccc}
\hline Localité & \multicolumn{3}{c}{ Aguié } & \multicolumn{3}{c}{ Ibohamane } \\
Variables & Pluie & NVDI_max & RUE & Pluie & NDVI_max & RUE \\
\hline Pluie & $\mathbf{1}$ & 0,35 & $\mathbf{- 0 , 5 2}$ & 1 & 0,11 & $\mathbf{- 0 , 4 5}$ \\
NVDI_max & 0,35 & $\mathbf{1}$ & 0,34 & 0,11 & 1 & $\mathbf{0 , 8 3}$ \\
RUE & $\mathbf{- 0 , 5 2}$ & 0,34 & $\mathbf{1}$ & $\mathbf{- 0 , 4 5}$ & $\mathbf{0 , 8 3 * *}$ & 1 \\
\hline
\end{tabular}

Légende : NDVI : Indice Normalisé de Végétation, RUE : Indice d'Efficacité de Pluie, ** corrélation significative au seuil de $1 \%$.

Tableau 5 : Paramètres statistiques globaux des trois variantes du NDVI situation d'Aguié.

\begin{tabular}{lcccccc}
\hline Localité & \multicolumn{3}{c}{ Aguié } & & \multicolumn{3}{c}{ Ibohamane } \\
\hline Paramètre & NDVI_thé & NDVI_obs & NDVI_rés & NDVI_th & NDVI_obs & NDVI_rés \\
\hline Maximum & 0,16 & 0,37 & $\mathbf{0 , 2 4}$ & 0,18 & 0,34 & $\mathbf{0 , 1 8}$ \\
Minimum & 0,10 & 0,14 & $\mathbf{0 , 0 4}$ & 0,11 & 0,12 & $\mathbf{0 , 0 1}$ \\
Moyenne & 0,13 & 0,33 & 0,20 & 0,14 & 0,26 & $\mathbf{0 , 1 2}$ \\
Ecart-type & 0,02 & 0,05 & 0,05 & 0,02 & 0,05 & 0,04 \\
CV (\%) & 11,76 & 14,64 & 22,92 & 0,18 & 21,26 & 36,86 \\
\hline NDVI_thé : NDVI théorique, NDVI_obs : NDVI observé, NDVI_rés : NDVI résidus, CV : Coefficient de variation.
\end{tabular}




\section{Cas d'Aguié}

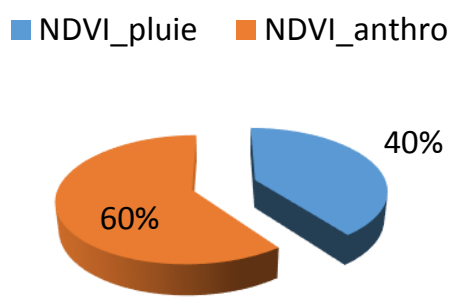

\section{Cas d'Ibohamane}

nNVI_pluie nNVI_anthro

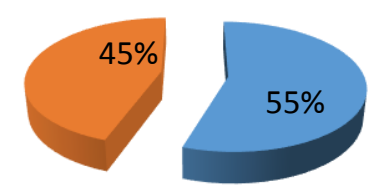

Figure 12 : Part anthropogénique versus climatique du reverdissement.

Tableau 6 : Valeurs R de Pearson et de p-values correspondantes entre les paramètres densité démographique et NDVI_max dans les deux communes.

\begin{tabular}{lcc}
\hline Localité & Aguié & Ibohamane \\
\hline Valeurs de R & 0,63 & 0,51 \\
p-values & 0,04 & 0,23 \\
\hline \multicolumn{2}{c}{ Légende : R coefficient de corrélation de Pearson. }
\end{tabular}

\section{DISCUSSION}

Principales caractéristiques des séries pluviométriques des deux communes

Cette étude a montré qu'il existe un faible niveau d'interdépendance entre pluies consécutives au cours des années récentes. Cela est vraisemblablement lié à l'intensification de la manifestation des aléas sur le régime pluvieux. La variabilité interannuelle des pluies enregistrée est beaucoup plus élevée dans le secteur aride d'Ibohamane par rapport au domaine semiaride d'Aguié. Panthou et al. (2014) avaient évoqué cette forte occurrence des phénomènes climatiques extrêmes dans la partie centrale du Sahel. Selon Ozer et al. (2010) cette forte variabilité peut être attribuée à deux causes principales qui agissent de façon conjointe. Dans un premier temps, la position assez irrégulière de la Zone de Convergence Intertropicale (ZCIT) favorise le développement d'orages convectifs ou organisés en lignes de grains entre les masses d'air équatorial et tropical. En second lieu, la manifestation de ces orages convectifs intervient tantôt en début, au milieu ou en fin de campagne agricole avec une cadence très variable sur l'échelle spatiale. C'est d'ailleurs ce qui explique la mauvaise distribution géographique des précipitations au Sahel. Les fortes oscillations indiquées par les anomalies pluviométriques enregistrées à Aguié et Ibohamane sont probablement une conséquence de ces phénomènes.

De plus, les résultats du test de Pettit en accord avec la segmentation de Hubert sur la série chronologique considérée montrent que l'évolution de la pluviométrie dans ces deux zones présente dans l'ensemble deux caractéristiques majeures. Une période sèche bien nette installée depuis 1980 devenue plus sévère entre 1984-1985 et qui a perduré beaucoup plus longtemps au niveau d'Ibohamane (zone aride) comparativement à Aguié (zone semi-aride). Un retour plus favorable de la pluviométrie lui succède à partir de 1997 avec cependant beaucoup de fluctuations. Le retour aux conditions climatiques favorables est intervenu nettement plus précocement à Ibohamane (année 1988) comparativement à Aguié où il a fallu attendre l'année 1994 pour avoir une tendance à l'amélioration de la pluviométrie. En revanche, dans le cas la situation d'Ibohamane, on a observé une persistance de la séquence. L'alternance des séquences sèches et humides est une constante plus ou moins bien établie au Sahel (Lebel et Ali, 2009). Cette étude a mis en évidence l'existence d'une période sèche et d'une période humide nettement bien 
démarquées autour des années 1990. En effet, Ozer et al. (2007) indiquaient la fin de cette tendance régressive du régime pluvieux autour des années 1990. Ces observations sont en accords avec les conclusions de cette étude qui positionnent les années de rupture entre 19931997 au test de Pettit. Les sécheresses enregistrées au cours des décennies 70-80 ne sont que des conséquences d'une tendance amorcée bien avant. L'amélioration récente et actuelle du régime pluviométrie au Niger a été évoquée par plusieurs auteurs parmi lesquels Hountondji et al. (2009) et Nicholson (2005).

\section{Liens entre tendance climatique et tendance au reverdissement}

Pour ces résultats, la diminution du RUE signifie que la pluviométrie a induit un couvert végétal faible à médiocre. Si en dépit de l'augmentation du NDVI, la valeur du RUE décline, on peut conclure que dans les deux cas l'amélioration de la couverture végétale n'est pas forcément une conséquence de la pluviométrie.

Selon Dardel et al. (2014), la tendance au reverdissement sahélien est définie par l'augmentation significative du NDVI observée depuis le début des années 80 . Ce phénomène est donc un fait assez bien évalué par télédétection (Herrmann et al., 2005 ; Hountondji, 2008). Bien que l'évolution du NDVI soit fluctuante d'une année à une autre dans le cas d'Aguié, globalement les résultats obtenus dans cette étude montrent une certaine tendance à une légère progression du NDVI sur le plan global. Cette faible augmentation globale ne saurait faire perdre de vue la forte variabilité spatiale de la manifestation du reverdissement sur le paysage dans les deux communes concernées par cette étude. La même tendance a été observée par Baggnian et al. (2013) dans la bande sud du Niger (Mardi et Zinder). De même Dardel et al. (2014) ont mis en évidence une tendance positive du NDVI sur une portion de l'Ouest Nigérien (le Gourma).

Cette étude a montré que la pluviométrie exprime une corrélation négative avec le NDVI. Aussi, plus le NDVI augmente plus le RUE diminue. Cela signifie que l'amélioration de la couverture végétale n'est pas le fait d'une augmentation de la pluviométrie. Ces résultats sont en accords avec ceux de Hountondji (2008) qui a enregistré une tendance stable à négative du RUE pendant que le NDVI augmente sur 68,4\% de 354 stations étudiées au Sahel.

Il faut souligner que ces résultats ont une portée locale puisque dans certains cas la sécheresse s'est traduite par une baisse de la productivité végétale comme cela a été enregistré par Sieza et al. (2019). Cet auteur utilisant l'indice de condition de la végétation et les valeurs de la pluviométrie annuelle de 1981 à 2017 a enregistré une dégradation de la biomasse consécutivement à cinq (5) poches pluviométriques.

\section{Liens entre dynamique anthropique et tendance au reverdissement}

Cette étude montre que là où la densité démographique est élevée le reverdissement est plus intense. En effet, sur la série 1977-2018, la densité démographique est nettement plus élevée à Aguié par rapport à Ibohamane de même que la tendance au reverdissement. C'est dire que mis à part les disparités liées à la nature des sols, à la forme du relief et aux conditions agro-techniques, la densité démographique se distingue comme facteur déterminant de la tendance au reverdissement. Des résultats similaires ont été obtenus par Mazzucato et Niemeijer (2001) dans le nord Burkina Faso, Mortimore (2005) à Machakos au Kenya et au Niger, Baggnian et al. (2012) dans le centre-Sud Maradi au Niger. La pratique généralisée de la Régénération Naturelle Assistée (RNA) peut être une des pistes pour expliquer ce lien étroit entre le reverdissement et la densité démographique. Des nombreux auteurs parmi lesquels Lawali et al. (2018), Morou et al. (2016), Larwanou et al. (2012) ont indiqué que la commune d'Aguié est une zone de prédilection de la pratique de la RNA. Cela soulève la nécessité de pousser des réflexions à petite échelle pour comprendre comment les populations des zones concentrées agissent dans le façonnement de leurs paysages à travers les pratiques de gestion durable des terres.

\section{Conclusion}

L'amélioration récente du régime pluviométrique prêtait à accorder une place importante à la dimension climatique dans l'explication du reverdissement sahélien. Cette étude avait donc pour objectif d'évaluer la part 
contributive de la dimension humaine dans ce processus. Elle a montré qu'un taux de $60 \%$ et 45\% du reverdissement est déterminé par le paramètre densité démographique respectivement dans la commune d'Aguié et d'Ibohamane. Cela permet de replacer le rôle central de la dimension anthropique dans la mutation paysagère récente de ces zones. Ces résultats impliquent que la promotion et la mise à l'échelle de la gestion durable des terres qui constitue la base du reverdissement doit nécessairement s'appuyer sur certains facteurs socioéconomiques déterminants qui influencent fortement cette dynamique. Et que des réflexions doivent être engagées pour mieux comprendre comment ces facteurs favorisent la tendance au reverdissement de ces zones.

\section{CONFLIT D'INTÉRÊTS}

Les auteurs déclarent qu'il n'existe aucun conflit d'intérêts pour cet article.

\section{CONTRIBUTIONS DES AUTEURS}

SS a collecté les données et a rédigé le premier draft du manuscrit. J-MKA a corrigé le manuscrit et le protocole du projet de cet article.

\section{REMERCIEMENTS}

Les auteurs de cet article adressent leurs remerciements à Monsieur Alfari Maiga du Centre Régional AGRHYMET de Niamey pour son appui dans l'acquisition des données pluviométriques et celles de l'indice NDVI nécessaires à la réalisation de cette étude.

\section{REFERENCES}

Ambouta J-M K, Amadou I, Souley I. 1998. Gestion de la fertilité et évolution des sols de Gakudi (Maradi, Niger). Cahiers Agricultures, 7 : 395-400.

Aubréville A. 1949. Climats, forêts et désertification de l'Afrique tropicale. Société d'édition de géographie coloniale et maritime, Paris, 351p. Available at http://www.frameweb.org/ev.php?ID=13 091_201\&ID2=DO_TOPIC

Baggnian I, Mohamadou A, Adamou M M, Lawali S, Adam T, Enfors E, Larwanou M, Tougiani A. 2012. Perceptions paysannes des tendances du reverdissement des zones dégradées au Niger. Université de Maradi. Journal des Sciences de l'Environnement, 1 (1) : 4352.

Baggnian I. 2014. Résilience des agroécosystèmes au Sahel: analyse du reverdissement dans le centre Sud du Niger. Thèse de Doctorat, option : sciences de l'environnement. Université Abdou Moumouni de Niamey. 184p.

Banque Mondiale. 2013. Evaluation des risques du secteur agricole au Niger: de la réaction aux crises à la gestion des risques à long terme. Rapport d'étude, $96 \mathrm{p}$.

Bégué A, Vintrou E, Ruelland D, Claden M, Dessay N. 2011. Can a 25-year trend in Soudano-Sahelian vegetation dynamics be interpreted in terms of land use change? A remote sensing approach. Global Environmental Change-Human and Policy Dimensions, 21 (2) : 413-420.

Buishand T A. 1982. Some methods for testing the homogeneity of rainfall records. Journal of Hydrology, 58 (1-2): 11-27. DOI: https://doi.org/10.1016/00221694(82)90066-x.

Brown LR, Wolf EC. 1985. Origins of the African Food Crisis. Challenge, Jan-Feb, p 50-52.

Dardel C, Kergoat L, Hiernaux P, Mougin E., Grippa M, Tucker CJ. 2014. Re-greening Sahel: 30 years of remote sensing data and field observations (Mali, Niger). Remote Sensing of Environment, 140 : 350 - 364. www.elsevier.com/locate/rse

Evans J, Geerken R. 2004. Discrimination between climate and human-induced dryland degradation. Journal of Arid Environments, 57: 535 - 554.

Fensholt R, Thomas T, Stisen S. 2006. Evaluation of AVHRR PAL and GIMMS 10-day composite NDVI time series products using SPOT-4 vegetation data for the African continent. International Journal of Remote Sensing, 27(13): 27192733.

DOI : https://doi.org/10.1080/01431160600567 761.

Garba A, Djima T I, Abdou L, Mahamane A. 2017 Caractérisation de la végétation ligneuse du bassin versant de la Maggia dans la commune rurale de Bagaroua (région de Tahoua). Int. J. Biol. Chem. 
Sci.,

11(2):

571-584.

http://ajol.info/index.php/ijbcs.

Gavaud M. 1968. Les sols bien drainés sur matériaux sableux du Niger. Essai de systématique régionale. Cah. ORSTOM, séri. Pédol., 6(3-4) : 278-307.

Habou AZ, Boubacar MK, Adam T. 2016. Les systèmes de productions agricoles du Niger face au changement climatique: défis et perspectives. Int. J. Biol. Chem. Sci., $\quad$ 10(3): $1262-1272$. http://ajol.info/index.php/ijbcs.

Herrmann SM, Anyamba A, Tucker CJ. 2005. Recent trends in vegetation dynamics in the African Sahel and their relationship to climate. Global Environmental Change Part A, 15: 394-404. DOI: https://doi.org/10.1016/j.gloenvcha.2005. 08.004.

Hountondji Y-C, Sokpon N, Ozer P. 2006. Analysis of vegetation trends using low resolution remote sensing data in Burkina Faso (1982_1999) for the monitoring of desertification. International Journal of Remote Sensing, 27(5): 871-884. DOI: https://doi.org/10.1080/01431160500382 782.

Hountondji Y-C, Sokpon N, Nicolas J, Ozer P. 2009. Ongoing desertification processes in the sahelian belt of West Africa, an evidence from the rain-use efficiency. Recent Advances in Remote Sensing and Geo-information, Processing for Land Degradation Assessment, Röder A, Hill J (eds). ISPRS Series, Taylor and Francis; 173-186.

Hubert P, Carbonnel JP, Chaouche A. 1989. Segmentation des séries hydrométéorologiques. Application à des séries de précipitations et de débits de l'Afrique de l'Ouest. Journal of Hydrology, 110 (3-4) : 349-367. DOI : https://doi.org/10.1016/00221694(89)90197-2.

Institut National de la Statistique du Niger. 2015. Etat et structures de la population du Niger en 2012. Pages 55. www.statniger.org.

Institut National de la Statistique du Niger. 2014. Répertoire National des Localités version 2014. Pages 719. www.statniger.org.
Lamb PJ. 1982. Persistence of sub-Saharan drought. Nature, 299, 46-48. https://doi.org/10.1038/299046a0.

Larwanou M, Moustapha AM, Rabe ML, Iro D. 2012. Contribution de la Régénération Naturelle Assistée des Ligneux dans l'approvisionnement en bois des ménages dans le département de Magaria (Niger) Int. J. Biol. Chem. Sci., 6(1): 24-36. http://ajol.info/index.php/ijbcs.

Larwanou M, Saadou M. 2010. The role of human interventions in tree dynamics and environmental rehabilitation in the Sahel zone of Niger. Journal of Arid Environments, $\quad \mathbf{7 5}$ : 194-200. www.elsevier.com/locate/jaridenv.6

Lawali S, Diouf A, Morou B, Abdou KK, Saidou L, Guero C, Mahamane A. 2018. Régénération Naturelle Assistée (RNA) : outil d'adoption et de résilience des ménages ruraux d'Aguié au Niger. Int. J. Biol. Chem. Sci., 12(1): 75-89. http://ajol.info/index.php/ijbcs.

Le Houérou HN. 1984. Rain Use Efficiency: a unifying concept in arid land ecology. Journal of Arid Environments, 7(3): 213247.

Lebel T, Ali A. 2009. Recent trends in the Central and Western Sahel rainfall regime (1990-2007). Journal of Hydrology, 375 : $52-64$.

Mahamane A. 2001. Usages des terres et évaluations végétales dans le département de Maradi. Dryland Research Working paper 27, Crewkerne, Somerset, Royaume-Uni, 44p.

Mazzucato V, Niemeijer D. 2001. Le Sahel : une dégradation des terres exagérée, un potentiel paysan sous-estimé Valentina $25 \mathrm{p}$.

Milich L, Weiss E. 2000a. GAC NDVI interannual coefficient of variation $(\mathrm{CoV})$ images: ground truth sampling of the Sahel along north-south transects. International Journal of Remote Sensing, 21: 235-260.

Milich L, Weiss E. 2000b. GAC NDVI images: relationship to rainfall and potential evaporation in the grazing lands of the Gourma (northern Sahel) and in the croplands of the Niger-Nigeria border (southern Sahel). International Journal of Remote Sensing, 21: 261-280. 
Morou B, Lawali S, Oumani A A, Ounani H, Guero C, Mahamane A. 2016. Ressources forestières ligneuses : diversité et usages dans le terroir villageois de Dan Saga. Afrique Science, 12(4): 228-239. http://www.afiquescience.info.

Mortimore M, Turner B. 2005. Does the Sahelian smallholder's management of woodland, farm trees, rangeland support the hypothesis of human-induced desertification? Journal of Arid Environments, 63: 567-595. www.elsevier.com/locate/jaridenv.

Nicholson SE. 2005. On the question of the "recovery" of the rains in the West African Sahel. Journal of Arid Environments, $\quad$ 63: 615-641. www.elsevier.com/locate/jaridenv

Olsson L, Eklundh L, Ardo J. 2005. A recent greening of the Sahel - trends, patterns and potential causes. Journal of Arid Environments, $\quad 63$ : 556566.www.elsevier.com/locate/jaridenv.

ORSTOM. 1964. Etude pédologique de l'Ader Doutchi, Rapport Général Centre ORSTOM de Dakar, No6500/129, République du Niger. 104p.

Ozer P, Gassani J, Hountondji Y-C, Niang A J, Ambouta J-M K. 2007. La désertification est-elle en recul au Sahel? Bois et Forêts des Tropiques, 293 (3).

Ozer P, Hountondji Y-C, Karimoune S, Manzo L O. Salmon M. 2010. Désertification au Sahel : Historique et Perspectives. BSGLg, 54 : 69-84. www.bsglg.be.

Panthou G, Vischel T, Lebel T. 2014. Recent trends in the regime of extreme rainfall in the central Sahel. International Journal of Climatology, 34: 3998-4006. DOI: 10.1002/joc.3984.

Pettit A N. 1979. A non parametric approach to the change-point problem. Royal Statistical Society, 28(2): 126-135. DOI: https://doi.org/10.2307/2346729.

Prince SD, De Colstroun EB, Kravitz LL. 1998. Evidence from rain-use efficiencies does not indicate extensive sahelian desertification, Global Change Biology, 4(4): 359-374.
Rasmussen K, Fog B, Madsen JE. 2001. Desertification in reverse Observations from northern Burkina Faso. Global Environmental Change, 11: 271-282. www.elsevier.com/locate/gloenvcha.

Raynaut C. 2001. Societies and nature in the Sahel: ecological diversity and social dynamics Université Bordeaux 2, 146 rue Le Saignat, 33076 Bordeaux, Cedex, France. Global Environmental Change, 11: 9-18. www.elsevier.com/locate/gloenvcha.

Reij C, Smaling EMA. 2005. Introduction: The "'Greening' of the Sahel. Journal of Arid Environments, 63: 535-537.

Reij C, Smaling EMA. 2005. Introduction: The "Greening" of the Sahel. Journal of Arid Environments, 63: 535-537.

Reij C, Smaling EMA. 2008. Analyzing successes in agriculture and land management in Sub-Saharan Africa: is macro-level gloom obscuring positive micro-level change? Land Use Policy, 25: 410- 420.

Seaquist JW, Hickler T, Ardö J, Heumann B W. 2009. Disentlangling the effects of climate and people on Sahel vegetation dynamics. Biogeosciences; 6: 469-477.

Seghieri J. 2017. Systèmes agroforestiers soudano-sahéliens. Tradition ou innovation? Institut de la Francophonie pour le Développement Durable, Liaison Energie-Francophonie, 105 : 92-96. www.ifdd.francophonie.org.

Sieza Y, Gomgnimbou APK, Serme I, Belem A. 2019. Etude de la variabilité climatique sur la dynamique d'occupation et d'utilisation des terres à des fins agropastorales dans la zone sud-soudanienne du Burkina Faso. Int. J. Biol. Chem. Sci., 13(4): 1980-1994. http://ajol.info/index.php/ijbcs.

Tougiani A, Guero C, Rinaudo T. 2009. Community mobilisation for improved livelihoods through tree crop management in Niger. Geo-Journal, 74(5) : 377 - 389 . 